\title{
THE ION CYCLOTRON, LOWER HYBRID AND ALFVEN WAVE HEATING METHODS
}

\author{
R. Koch \\ Laboratory for Plasma Physics \\ Ecole Royale Militaire - Koninklijke Militaire School \\ B-1000 Brussels, Belgium
}

\begin{abstract}
This lecture covers the practical features and experimental results of the three heating methods. The emphasis is on ion cyclotron heating. First, we briefly come back to the main non-collisional heating mechanisms and to the particular features of the quasilinear coefficient in the ion cyclotron range of frequencies (ICRF). The specific case of the ion-ion hybrid resonance is treated, as well as the polarisation issue and minority heating scheme. The various ICRF scenarios are reviewed. The experimental applications of ion cyclotron resonance heating (ICRH) systems are outlined. Then, the lower hybrid and Alfvén wave heating and current drive experimental results are covered more briefly. Where applicable, the prospects for ITER are commented.
\end{abstract}

\section{ION CYCLOTRON HEATING}

\section{I.A. Introduction}

Before going in further details of heating mechanisms, it is important to recapitulate the order of magnitude of the different quantities characterising the plasma and the RF. A table of plasma parameters (Table $1)$, typical of moderate plasma performance in a machine like JET, was given earlier ${ }^{1}$. Two important parameters that characterise the collisionality of the plasma are to be added to complete the picture: the ion an electron collision frequencies: $v_{e} \approx 10 \mathrm{kHz}, v_{i} \approx 100 \mathrm{~Hz}$. A JET-type machine is characterised by the following parameters:

$R_{0}=3 \mathrm{~m}, 2 \pi R_{0} \approx 20 \mathrm{~m} ; a_{p}=1.5 \mathrm{~m}, 2 \pi a_{p}=10 \mathrm{~m}$.

In the light of these numbers, one sees that the time for a cyclotron gyration is extremely short: $10 \mathrm{ps}$ for an electron, $40 \mathrm{~ns}$ for an ion. During this single gyration, the electron travels $0.4 \mathrm{~mm}$ in the toroidal direction and the ion $2 \mathrm{~cm}$. It takes $1 \mu$ s to an electron to complete a toroidal turn around the machine, $40 \mu$ s to an ion. During this turn, an electron has performed 50,000 cyclotron gyrations, an ion 1,000. This means that gyro motion is an extremely fast process as compared to transit times across any macroscopic area. Equivalently, the gyro radii of electrons $(0.05 \mathrm{~mm})$ and of ions $(3 \mathrm{~mm})$ are small as compared to plasma size. The plasma is nearly non-collisional: the electron mean free path is $3 \mathrm{~km}$ and the ion one $5 \mathrm{~km}$, or, respectively, 150 and 250 toroidal revolutions.

The following parameters are typical of an ICRF system:

- frequency: $\quad \mathrm{f} \approx 10-100 \mathrm{MHz}$

- Power: $2 \mathrm{MW} / \mathrm{antenna} \mathrm{strap}$

- Voltage: $10-50 \mathrm{kV}$ at the antenna

- Antenna current: $I_{A} \approx 1 \mathrm{kA}$

- Central conductor: width $\approx 0.2 \mathrm{~m}$, length $\approx 1 \mathrm{~m}$, distance to the plasma $5 \mathrm{~cm}$, to the wall $20 \mathrm{~cm}$

- Typical RF electric field: $20 \mathrm{kV} / \mathrm{m}$

- Typical RF magnetic induction: $10^{-3} \mathrm{~T}$

ICRF antennas are quite often built as boxes enclosing one or several central conductors to whom the high voltage is applied. Such a central conductor is also called a strap. The maximum voltage that could be applied to an antenna operating in a tokamak, in the presence of plasma, lies around $45 \mathrm{kV}$. The ratio of voltage to current and the power coupling capability of an antenna are determined by the geometry of the antenna and the plasma edge properties $^{1}$. The electric field at the antenna is easily evaluated by dividing the input voltage by the antenna length. This is valid for the field component polarised along the antenna; other field components can be much higher in the vicinity of the antenna feeding point. Except for possible focusing effects, the field inside the plasma is lower than at the antenna, because $k_{\perp}|E|^{2}$ is nearly constant and $k_{\perp}$ is roughly proportional to $\sqrt{n}$. The typical RF magnetic field is easily computed using ampere's law: $2 \pi d H=I_{A}$ where $d$ is typical of the distance over which the magnetic field can spread. These electrostatic- and magnetostatic-type estimates have a meaning due to the fact that the vacuum wavelength is large as compared to the antenna dimensions: 
$\lambda_{0}=c / f \approx 10 \mathrm{~m}(30 \mathrm{MHz})$.

\section{I.B. Linearity}

These numbers show that the RF causes only a small perturbation of the particle trajectory. First, the RF magnetic field is much smaller than the static one:

$B_{\mathrm{RF}} \approx 10^{-3} \mathrm{~T} \ll B_{0} \approx 3 \mathrm{~T}$.

Second, the RF electric field $\approx 20 \mathrm{kV} / \mathrm{m}$ is also much smaller than the $\mathbf{v} \times \mathbf{B}$ field associated with the ion's (and even more electron's) thermal motion: $V_{\mathrm{ti}} \times B_{0} \approx 1.5 \mathrm{MV} / \mathrm{m}$. Third, we shall show below that the perturbation of the parallel motion is also small. Let us write the equation of motion of a particle in the RF field, decomposing the motion into an unperturbed (thermal) part labelled 0 and a perturbed part: $\mathbf{v} \rightarrow \mathbf{v}_{0}+\mathbf{v}$

$m\left(\frac{d \mathbf{v}_{0}}{d t}+\frac{d \mathbf{v}}{d t}\right)=Z e\left(\mathbf{E}+\mathbf{v}_{0} \times \mathbf{B}_{0}+\mathbf{v}_{0} \times \mathbf{B}+\mathbf{v} \times \mathbf{B}_{0}+\mathbf{v} \times \mathbf{B}\right)$

Subtracting the unperturbed part of this equation

$m \frac{d \mathbf{v}_{0}}{d t}=Z e \mathbf{v}_{0} \times \mathbf{B}_{0}$

which describes the unperturbed cyclotron motion leaves us with the perturbed part of the equation of motion.

$m \frac{d \mathbf{v}}{d t}=Z e\left(\mathbf{E}+\mathbf{v}_{0} \times \mathbf{B}+\mathbf{v} \times \mathbf{B}_{0}+\mathbf{v} \times \mathbf{B}\right)$

In the r.h.s parenthesis the last term is clearly negligible as compared to the 3rd one. We thus arrive at the following equation, which is linear in the perturbed field amplitude:

$m \frac{d \mathbf{v}}{d t}=Z e\left(\mathbf{E}+\mathbf{v}_{0} \times \mathbf{B}+\mathbf{v} \times \mathbf{B}_{0}\right)$

Finally, we can estimate the correction to the parallel uniform motion due to the RF field. Taking the parallel component of Eq.(3) and $d / d t \approx \omega_{\mathrm{ci}}$, we get for an ion

$m \omega_{c} v_{/ /}=Z e\left[E_{/ /}+\left(\mathbf{v}_{0} \times \mathbf{B}\right)_{/ /}\right]$

or, in order of magnitude

$v_{/ /} \approx\left|\frac{E_{/ /}}{B_{0}}\right| \quad$ or $\quad \approx V_{t i}\left|\frac{B_{R E}}{B_{0}}\right|$

Thus, in any case the RF-induced particle quiver velocity is small as compared to the thermal (unperturbed) ion velocity. In summary, the corrections to the particle's trajectory due to the RF field are indeed small, and the linearisation is justified.

Although Eq.(4) is linear in the fields, it is not at all linear for the unknowns $\mathbf{r}$ and $\mathbf{v}$. Indeed, the electric field depends non-linearly on the particle position $\mathbf{r}$. However, we have seen that the RF fields only cause small perturbations to the particle trajectories. Therefore, we can neglect these small deviations in the evaluation of the electromagnetic field at the particle location and write $\mathbf{E}(\mathbf{r}) \approx \mathbf{E}\left(\mathbf{r}_{0}\right) ; \quad \mathbf{B}(\mathbf{r}) \approx \mathbf{B}\left(\mathbf{r}_{0}\right)$ with $\mathrm{d} \mathbf{r}_{0} / \mathrm{dt}=\mathbf{v}_{0}$ and $\mathbf{v}_{0}$ is the solution of the unperturbed equation of motion Eq.(2). Then the equation determining the velocity perturbation is

$$
m \frac{d \mathbf{v}}{d t}=Z e\left[\mathbf{v} \times \mathbf{B}_{0}+\mathbf{E}\left(\mathbf{r}_{0}\right)+\mathbf{v}_{0} \times \mathbf{B}\left(\mathbf{r}_{0}\right)\right]
$$

This is now a linear equation that can be solved explicitly if the unpertubed trajectories $\left(\mathbf{r}_{0}, \mathbf{v}_{0}\right)$ are known explicitly. The next step in the solution of the problem is to decompose the zero-th order motion into a guiding centre motion $\mathbf{r}_{\mathrm{G}}$ and a gyromotion $\boldsymbol{\rho}$ :

$\mathbf{r}_{0}=\mathbf{r}_{\mathrm{G}}+\boldsymbol{\rho}$

and to expand around the guiding centre motion:

$\mathbf{E}\left(\mathbf{r}_{0}\right)=\sum_{n=0}^{\infty} \frac{\left[(\rho . \nabla)^{n} \mathbf{E}\right]_{0}=\mathbf{r}_{G}}{n !}$

This procedure is known as the small Larmor radius expansion. In general geometry this development can become quite heavy ${ }^{2}$, but in straight geometry, it can be performed explicitly. In the latter case (uniform plasma), the decomposition of the motion, Eq.(9) is exact and the expansion Eq.(10) can be expressed in terms of Bessel functions, see Eq. $(13,16)$ of ref. ${ }^{3}$. Like in the case of Landau damping $^{1}$, the explicit integration of the linearised equation of motion Eq.(8) can, in some cases, lead to secular solutions, corresponding to resonant denominators in Fourier space ${ }^{4}$. The same denominators are found back under the velocity integral in the expression of the full hot conductivity tensor ${ }^{3}$, leading to the general resonance condition

$\omega-n \omega_{c}-k_{/ /} \nu_{/ /}=0 ; \quad n=0, \pm 1, \pm 2, \ldots$

\section{I.C. The cyclotron absorption mechanisms}

\section{I.C.1 Resonances due to non-rotating fields}

If we look at the $n=0$ contribution to the conductivity tensor Eq.(20) in ref. ${ }^{3}$, we see that only the lower right $2 \times 2$ part of the $S_{\mathrm{n}}$ matrix is non-zero. For a uniform electric field $\left(k_{\perp} \mathbf{E}=\partial \mathbf{E} / \partial x=0\right)$, only the $z z$ term survives. It correspond to resonant parallel acceleration by the parallel electric field, i.e. to Landau damping. The three other terms require, at least, a gradient of the electric field $(\partial \mathbf{E} / \partial x \neq 0)$. By computing the expression $\mathbf{E}^{*} \cdot \boldsymbol{\sigma} . \mathbf{E}$, (proportional to the absorbed wave power) for the case where only $E_{\mathrm{y}}$ is present, it is easy to see that this quantity is proportional to

$\left(k_{\perp} E_{\mathrm{y}}\right)^{2}=\left(\omega B_{\mathrm{z}}\right)^{2}$

If we assume $E_{\mathrm{y}}$ to be linear, we thus have a case of acceleration by a uniform $B_{\mathrm{z}}$ field propagating in the $\mathrm{z}$ direction with $\omega=k_{/ /} v_{/ /}$. We have seen ${ }^{1}$ that the resonance mechanism in this case is transit time magnetic pumping (TTMP). More detail about this and about the interplay between Landau damping and TTMP in the case both $E_{\mathrm{y}}$ and $E_{\mathrm{z}}$ differ from zero, can be found in the book of Stix ${ }^{5}$. 
Landau damping and TTMP are not important mechanisms for the ions in the ICRF because $k_{/ /} V_{\text {th }}$ is usually much smaller than $\omega \approx \omega_{\mathrm{ci}}$, as can be seen from Table 1 and $k_{/ /}$estimates ${ }^{1}$. For the electrons, on the contrary, they are the only damping mechanisms in this frequency range. As they correspond to parallel acceleration, they are of primary importance for the fast wave current drive applications.

I.C.2. Resonances due to the left-handed component of the field.

This is the case where the wave has the same handedness as the ions and the resonances correspond to $n>0$ in Eq.(11). If $n=1$, we have the case of the fundamental cyclotron resonance, which was discussed already $^{1}$ :

$\omega=\omega_{c}+k_{/ /} v_{/ /}$

Because the Doppler shift $k_{/ /} v_{/ /}$is small, this requires that the operating frequency be rather close to the ion cyclotron frequency. Looking at the expression of $S_{\mathrm{n}}$ in ref. $^{3}$, we see that such a resonance can be caused by a uniform field $\left(k_{\perp} \mathbf{E}=\partial \mathbf{E} / \partial x=0\right)$. On the contrary, for second harmonic damping $(n=2)$ to exist, the same expression shows that a gradient of the electric field is required. Similarly, higher harmonic damping requires the existence of non-vanishing higher derivatives of the electric field.

While it is easy to imagine how the fundamental cyclotron resonance works, it is much harder to visualise the reason why a particle rotating at the frequency $\omega_{\mathrm{c}}$ can resonate with a wave that is rotating at twice this frequency! In order to understand how this happens it is useful to come back first to the fundamental resonance and look at the simpler situation where the wave propagates (or is non-uniform) in the $\mathrm{x}$-direction while the E-field is polarised in the y-direction. The principle is shown on top of Fig.1. The particle travels from left to right and at the initial moment $t=0$, I assume that the electric force is in phase with the particle velocity. After a quarter period, if the field oscillates at the cyclotron frequency, $\omega=\omega_{\mathrm{c}}$, the field is zero, after half a period it is negative, etc. Therefore, either the force is in phase with the particle motion, or it is zero and over one cyclotron period, the particle experiences a net acceleration in the direction of its motion. If, with the same uniform field distribution, the field varies at twice the cyclotron frequency $\omega=2 \omega_{\mathrm{c}}$, after a quarter period, it has changed sign, points in the negative $y$ direction and is perpendicular to the particle's velocity. After half a cyclotron period, its phase has varied of $2 \pi$, it is again positive, points out of the paper in the direction opposite to the particle's velocity and so on. Hence, over a cyclotron period, the average force exerted on the particle is zero, there is no net acceleration and no resonance.
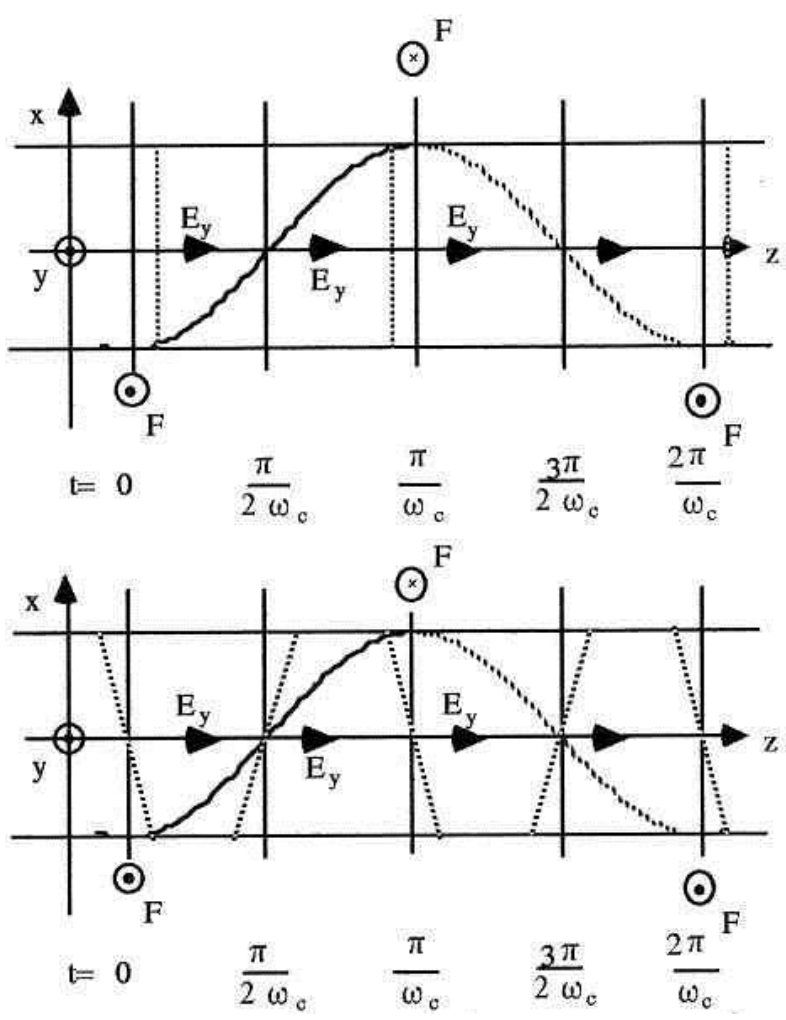

Fig. 1. Mechanism of the fundamental (top) and second harmonic (bottom) resonance. The ion moves in the z-direction and the projection of its helical motion on the $(x, z)$ plane is shown: solid line, above the plane; dotted line, below. The electric field distribution along $x$ is shown at quarter periods of the cyclotron frequency. The direction of the electric force $F$ at the particle's position is also indicated.

However, if the field varies linearly in space, as shown on the bottom of Fig.1, at the same time as it alternates sign, the particle moves from one side of the gradient to the other, therefore keeping in phase with the electric force and experiencing a net acceleration over one period, as shown. It is therefore the interplay between the field periodicity and the sampling of the field non-uniformity by the particle that allows resonance at the cyclotron harmonics.

\section{I.C.3. Resonances due to the right-handed component of} the field.

This type of resonance, corresponding to $n<0$, is somewhat counter-intuitive as the field rotates in the direction opposite to the particle. However the resonance condition shows that, in order to come into resonance, the particle must have a very large velocity:

$v_{/ /}=\frac{\omega+|\eta| \omega_{c}}{k_{/ /}}>V_{t i}$ 
One can then understand the resonance process as follows. Let us start with a particle with zero parallel velocity and a right-hand polarised field at frequency $\omega$ slightly larger than $\omega_{\mathrm{ci}}$ and positive phase velocity $\omega / k_{/ /}>0$. The particle sees the field rotating slightly faster than itself, in the opposite direction. Then if the particle starts to move at a speed $v_{/ /}>0$, in the reference frame moving at the same velocity, the field is now rotating at the slightly lower frequency $\omega=\omega-k_{/ /} v_{/ /}$. If $v_{/ /}$is large enough, this frequency may equal $\omega_{\mathrm{ci}}$ but the ion and the field are rotating in opposite directions. Then, if the particle's velocity becomes much larger, the field may become stationary $\omega-k_{/ /} v_{/ /}=0$ : the particle is gyrating in a stationary field. This cannot give rise to a resonance. If $v_{/ /}$is still increased, $\omega-k_{/ /} v_{/ /}<0$ and the wave field will start to rotate in the left-handed direction. Still increasing the particle's velocity, we can come to the situation where $\omega-k_{/ /} v_{/ /}=-\omega_{\mathrm{ci}}$ which means that the field is left-hand polarised and rotating at the cyclotron frequency, thereby causing resonant acceleration. This absorption mechanism is generally negligible for particles belonging to the thermal population in the plasma. However, for the much faster particles belonging to a high-energy tail, either created by the RF itself, or by NBI or for $\alpha$-particles, this mechanism may not be negligible at all. Even more so, as we shall see, as the right-hand field component tends to dominate in the fast wave, in the vicinity of resonances.

\section{I.C.4. Quasilinear diffusion coefficient}

Ion cyclotron heating tends to create high-energy tails in the distribution function of the heated particles. For heating at the $n$-th cyclotron harmonic, the diffusion coefficient is of the form:

$D \propto\left|J_{n-1} E_{+}+J_{n+1} E\right|^{2} \approx\left|J_{n-1}\left(\frac{k_{\perp} v_{\perp}}{\omega_{c}}\right)\right|^{2}\left|E_{+}\right|^{2}$

where $E_{+}$and $E_{\text {- }}$ are, respectively, the left-hand and righthand components of the electric field:

$E_{+}=\frac{1}{2}\left(E_{x}+i E_{y}\right) ; \quad E_{-}=\frac{1}{2}\left(E_{x}-i E_{y}\right)$

One notices the presence of a right hand component contribution in the diffusion coefficient. For not too fast particles in the FW field, this contribution is negligible because the argument of the Bessel functions is small. This is not necessarily the case for fast ions. In the case of the thermal population, one can take only the first term of the power series expansion of the Bessel functions, and we get:

- Fundamental: $D \propto K\left|E_{+}\right|^{2}$ with $K$ a constant

- Second harmonic: $D \propto\left|\frac{k_{\perp} v_{+}}{\omega_{c}}\right|^{2}\left|E_{+}\right|^{2}$
- n-th harmonic: $D \propto\left|\frac{k_{\downarrow} v_{+}}{\omega_{c}}\right|^{2(n-1)}\left|E_{+}\right|^{2}$

A first conclusion to be drawn from these expressions is that, as $k_{\perp} r_{\mathrm{L}}$ is a small quantity, the diffusion coefficient (hence the strength of the heating) strongly decreases with the harmonic number. Second, fundamental heating pushes all particles with the same strength irrespective of their velocity: the diffusion coefficient is independent of velocity. On the contrary, harmonic heating tends to accelerate more the faster particles. A consequence of this last observation is that harmonic heating tends to create tails at higher energy than fundamental heating. In the same vein, harmonic heating tends to interact more with faster particles like beams or alphas.

\section{I.D. The FW dispersion and polarisation}

The FW equation was given in section II.C of ref. ${ }^{1}$. We shall now specialise the expressions of the dielectric tensor components ${ }^{3}$ to the ICRF where

$\omega \approx \omega_{\mathrm{ci}} \ll \omega_{\mathrm{pi}} \ll \omega_{\mathrm{pe}}, \omega_{\mathrm{ce}}$,

We obtain

$$
\begin{aligned}
S & =1-\frac{\omega_{p e}^{2}}{\omega^{2}-\omega_{c e}^{2}}-\sum_{i} \frac{\omega_{p i}^{2}}{\omega^{2}-\omega_{c i}^{2}} \approx-\sum_{i} \frac{\omega_{p i}^{2}}{\omega^{2}-\omega_{c i}^{2}} \\
D & =\frac{\omega_{c e}}{\omega} \frac{\omega_{p e}^{2}}{\omega^{2}-\omega_{c e}^{2}}+\sum_{i} \frac{\omega_{c i}}{\omega} \frac{\omega_{p i}^{2}}{\omega^{2}-\omega_{c i}^{2}} \\
& \approx \frac{\omega_{p e}^{2}}{-\omega \omega_{c e}}+\sum_{i} \frac{\omega_{c i}}{\omega} \frac{\omega_{p i}^{2}}{\omega^{2}-\omega_{c i}^{2}}=\sum_{i} \frac{\omega}{\omega_{c i}} \frac{\omega_{p i}^{2}}{\omega^{2}-\omega_{c i}^{2}}
\end{aligned}
$$

The first line of Eq.(12) of ref. ${ }^{1}$ gives the relation linking $E_{\mathrm{x}}$ and $E_{\mathrm{y}}$ and can be used to compute the ratio of the leftto right-hand components of the electric field:

$\left|\frac{E_{x}+i E_{y}}{E_{x}-i E_{y}}\right|=\left|\frac{D+\left(S-N_{/ /}^{2}\right)}{D-\left(S-N_{/ /}^{2}\right)}\right|$

Let us now consider the case of a single ion species. Eqs.(18-19) become

$$
S \approx-\frac{\omega_{p i}^{2}}{\omega^{2}-\omega_{c i}^{2}} ; D \approx-\frac{\omega}{\omega_{c i}} S
$$

and

$\left|\frac{E_{x}+i E_{y}}{E_{x}-i E_{y}}\right| \approx\left|\frac{1-\frac{\omega}{\omega_{c i}}-N_{/ /}^{2} / S}{1+\frac{\omega}{\omega_{c i}}-N_{/ /}^{2} / S}\right| \approx\left|\frac{\omega-\omega_{c i}}{\omega+\omega_{c i}}\right|$

The last approximate equality follows from

$$
N_{/ /}^{2} / S \approx \frac{N_{/ /}^{2}\left(\omega_{c i}^{2}-\omega^{2}\right)}{\omega_{p i}^{2}}<<1
$$

The striking feature of the result Eq.(22) is that, at cyclotron resonance $\omega=\omega_{\mathrm{ci}}$, in a plasma with only one ion species $i$, the left-hand polarised component of the wave vanishes. Thus the cyclotron resonance mechanism cannot 
work because the resonant wave component is absent! Incidentally, this shows explicitly why there is no singularity of the fast wave propagation at the cyclotron frequency $^{3}$ : the resonant wave component is blocked by the resonance and all the power is transferred to the other component. This dramatic result can be avoided by heating a small amount of ions of one species in a plasma with ions of another species. This is called the minority heating scenario. Consider, for example a plasma of deuterium with a minority of hydrogen. Then the wave polarisation is determined by the majority component while the wave frequency is the cyclotron frequency of hydrogen. Inserting $\omega=\omega_{\mathrm{cH}}=2 \omega_{\mathrm{cD}}$ in Eq. (22) yields

$\left|\frac{E_{x}+i E_{y}}{E_{x}-i E_{y}}\right| \approx\left|\frac{\omega-\omega_{c D}}{\omega+\omega_{c D}}\right|=\left|\frac{2 \omega_{c D}-\omega_{c D}}{2 \omega_{c D}+\omega_{c D}}\right|=\frac{1}{3}$

This is the most widely used minority heating scenario, denoted D-(H) to indicate that a minority of $\mathrm{H}$ is heated in a majority $\mathrm{D}$ plasma. This concept of heating a given species in a mixture can be extended to other mixtures. For example in a reactor, one can heat $\mathrm{D}$ at the fundamental in a D-T mixture. Then, for not too large D concentration, the ratio of polarisations is:

$\left|\frac{E_{x}+i E_{y}}{E_{x}-i E_{y}}\right| \approx\left|\frac{\omega_{c D}-\omega_{c T}}{\omega_{c D}+\omega_{c T}}\right|=\frac{1}{5}$

This explanation has the advantage of simplicity and to some extent gives a good picture of reality. The shrewd reader would however notice by working out himself the complete polarisation expression for a mixture of ions that even in this case the left-handed polarisation vanishes at each cyclotron harmonic! The final explanation can only be obtained by taking hot-plasma (absorption) effects ${ }^{3}$ into consideration and noting that in a mixture the wave lefthanded component has a significant magnitude over a much wider region around the cyclotron harmonic than in the single-ion case. This is of course particularly evident when only a small minority is considered. This allows non-collisional damping at Doppler-shifted frequencies to remain efficient over a much wider range as compared to the single-ion case.

Another way to avoid the polarisation problem is to work at harmonics of the cyclotron frequency. Then

$\left|\frac{E_{x}+i E_{y}}{E_{x}-i E_{y}}\right| \approx\left|\frac{n \omega_{c i}-\omega_{c i}}{n \omega_{c i}+\omega_{c i}}\right|=\frac{n-1}{n+1}$

however, as we saw in Eq.(16.3), the damping strength strongly decreases with harmonic number.

\section{I.E. The ion-ion hybrid resonance}

Let us now consider, like in ref. ${ }^{3}$, the case of a $50 \%$ $50 \%$ mixture of $\mathrm{D}$ and $\mathrm{T}$.
$n_{\mathrm{D}}=n_{\mathrm{T}}=n_{\mathrm{e}} / 2$

Then, taking into account the fact that $\omega_{\mathrm{cD}}=\omega_{\mathrm{cH}} / 2$ and $\omega_{\mathrm{cT}}$ $=\omega_{\mathrm{cH}} / 3$, and defining

$\omega_{p H}=\sqrt{2} \omega_{p D}=\sqrt{3} \omega_{p T}$

we can recast the expressions for $S$ and $D$, Eq.(18-19) as

$S=\frac{2 \omega_{p H}^{2}}{\omega_{c H}^{2}-4 \omega^{2}}+\frac{3 \omega_{p H}^{2}}{\omega_{c H}^{2}-9 \omega^{2}}=\frac{5 \omega_{p H}^{2}\left(\omega_{c H}^{2}-6 \omega^{2}\right)}{\left(\omega_{c H}^{2}-4 \omega^{2}\right)\left(\omega_{c H}^{2}-9 \omega^{2}\right)}(26.1)$

$D=\frac{\omega}{\omega_{c H}}\left[\frac{4 \omega_{p H}^{2}}{\omega_{c H}^{2}-4 \omega^{2}}+\frac{9 \omega_{p H}^{2}}{\omega_{c H}^{2}-9 \omega^{2}}\right]$

Eq.(26.1) clearly shows that $S$ will vanish -which is the condition of wave resonance for perpendicular propagation $^{3}$ - when $\omega_{c H}=\sqrt{6} \omega$. This is the ion-ion hybrid resonance condition for a $50 \%-50 \%$ D-T plasma. One can likewise obtain this resonance condition from the FW wavevector Eq.(14.2) of ref. ${ }^{1}$, for the case of purely perpendicular propagation $\left(k_{/ /}=0\right)$ :

$k_{\perp F W}^{2}=k_{0}^{2} S-\frac{\left(k_{0}^{2} D\right)^{2}}{k_{0}^{2} S}=k_{0}^{2} \frac{5 \omega_{p H}^{2}}{\omega_{c H}^{2}} \frac{\left(\omega_{c H}^{2}-5.76 \omega^{2}\right)}{\left(\omega_{c H}^{2}-6 \omega^{2}\right)}$

Note that the general $\left(k_{/ /} \neq 0\right)$ resonance condition of the $\mathrm{FW}$ is

$k_{0}^{2} S-k_{/ /}^{2}=0$

and is not one of the general resonances $A=0$ discussed in ref. ${ }^{3}$. In fact, it does not exist in the full cold-plasma dispersion relation, where a conversion between the FW and the slow wave (Eq.(62) of ref. ${ }^{3}$ ) takes place instead of a resonance. The FW resonance Eq.(28) arises because of the neglect of the slow wave.

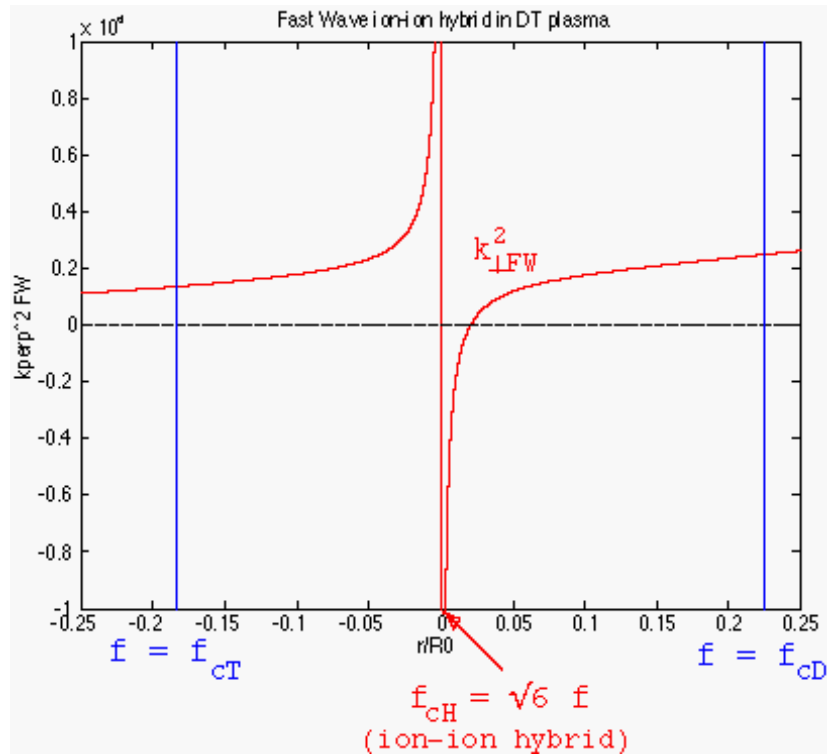

Fig. 2. Variation of the FW's wavevector(squared) over the plasma cross-section. The plasma density is assumed uniform. The ion-ion hybrid is located in the centre $x=0$. 
We are now in a position to show that the model equation Eq.(38) of ref. ${ }^{1}$ is a reasonable simplified resonance model. For the specific case of the ion-ion hybrid, and using Eq.(27) for simplicity, we shall also be able to show how to determine which sign to use in Eq.(43) of ref. ${ }^{1}$. In a tokamak, the toroidal magnetic field decreases as $1 / R, R$ the major radius. Accordingly, the FW's wavevector varies across the plasma. Such a variation is shown in Fig.2, in the vicinity of the ion-ion hybrid resonance and assuming that the plasma density is constant for simplicity. Let us define the resonance location as $R=R_{0}$ and

$\omega_{c H 0}^{2}=6 \omega^{2} ; \quad \omega_{c H}=\frac{\omega_{c H 0} R_{0}}{R}=\frac{\omega_{c H 0} R_{0}}{R_{0}+x}$

With these definitions, we can re-write the resonant denominator

$\omega_{c H}^{2}-6 \omega^{2}=\frac{\omega_{c H 0}^{2} R_{0}^{2}}{\left(R_{0}+x\right)^{2}}-6(\omega+i v)^{2}$

where we have introduced a small imaginary part to the frequency, in agreement with the causality rule ${ }^{3}$. In the vicinity of the resonance $(x / R \ll 1)$,

$$
\begin{aligned}
& \omega_{c H}^{2}-6 \omega^{2} \approx-\frac{12 \omega^{2}}{R_{0}}\left(x+\frac{i v}{\omega} R_{0}\right) \\
& k_{\perp F W}^{2} \approx-\frac{k_{0}^{2} \omega_{p H}^{2}}{60 \omega^{2}} \frac{R_{0}}{\left(x+i \frac{v}{\omega} R_{0}\right)}
\end{aligned}
$$

This proves our statements, as the singularity is indeed $1 / x$ and the pole is slightly below the real $x$-axis. It is thus circled clockwise while performing the $x$-integration in Eq.(42) of ref. ${ }^{1}$, which implies that the negative sign has to be taken in Eq.(43) of ref. ${ }^{1}$, and that, given the sign in the r.h.s. of Eq.(31.2), the wave energy is indeed absorbed rather than emitted- at the crossing of the resonance.

\section{I.F. Scenarios}

In present-day machines, the most usual scenario is fundamental heating of a hydrogen minority in a D plasma, or $\mathrm{D}-(\mathrm{H})$. Other mixtures have been tried successfully, like $\mathrm{D}-\left({ }^{3} \mathrm{He}\right)$, or even heavy minority scenarios like $\mathrm{H}-(\mathrm{D}), \mathrm{H}-\left({ }^{3} \mathrm{He}\right)$. Second and third harmonic heating, like $f=2 f_{\mathrm{cH}}$ or $f=3 f_{\mathrm{cD}}$, have been used, either as a majority or as a minority scenario. Mode conversion heating was proven to heat efficiently electrons, and FW current drive based on Landau-TTMP damping has been shown to follow theoretical expectations. In D-T plasmas, nearly all possible scenarios have been tested, T-(D), D-(T), second harmonic T, mixed $f=2 f_{\mathrm{cT}}$ and fundamental minority ${ }^{3} \mathrm{He}$, D-T- $\left({ }^{3} \mathrm{He}\right)$ and even mode conversion. Finally, let us note that direct ion Bernstein wave launch experiments have also been performed, with limited success in terms of power coupling, but inducing in some cases poloidal rotation and transport barrier formation. A good survey of these experiments, together with a large number of references is given in ref. ${ }^{6}$.

\section{I.G. Database and applications}

High power ICRH systems have been installed in a large number of machines. A maximum power of $22 \mathrm{MW}$ was coupled to the plasma in $\mathrm{JET}^{7}$. ICRH power was injected in various sorts of plasmas, including L-mode, ELM-free and ELMy H-mode, RI-mode ${ }^{8}$. In D-T, a record steady-state fusion $Q$ performance $(Q=0.22)$ was achieved with ICRH alone in $\mathrm{JET}^{9}$. At the occasion of the compilation of transport databases for ITER, it was noted that no significant differences in heating efficiency can be observed between the RF (ICRH and ECRH) subset and the rest of the database ${ }^{10}$. Although it is generally the case that the heating efficiency is equivalent for all auxiliary heating methods, there are cases where differences are observed. They are usually interpreted as resulting either from differences in power deposition profile or from a different impact on confinement. As an example of the latter, the choice of heating method - H/D co/counter NBI / ICRH- D-injection, can have definite impact on the achievement and quality of the RI-mode on TEXTOR ${ }^{11}$.

Like in the case of NBI, the neutron production rate furnishes an indirect measurement of the tail created by the RF. Comparisons with theoretical predictions indicate that the tail formation process is fairly well understood ${ }^{12}$. Fast ion energy content ${ }^{13}$ and direct tail measurements ${ }^{14}$ provide similar indications. Other comparisons have addressed the question of ion/electron power partition and power deposition profile ${ }^{15,16}$. The interaction of ICRH with beam-injected fast-ions was also investigated, in particular in relation with third harmonic heating and RFinduced fast particle diffusion ${ }^{17}$. Good agreement with theoretical predictions was observed. FW electron current drive has been tested in DIII-D and Tore-Supra, showing good agreement with the expected $T_{\mathrm{e}}$-scaling ${ }^{6}$. Minorityion current drive, by flattening or steeping the current density profile allowed control of the sawtooth frequency ${ }^{18}$. ICRF systems have recently found new applications in the field of plasma production in presence of a static magnetic field. The plasmas produced in this way could be used for efficient wall conditioning or for start-up assistance ${ }^{11,19,20}$.

An ICRF system has been designed for ITER ${ }^{6}$, which would couple $50 \mathrm{MW}$ through three ports. A rather large voltage is required $(42 \mathrm{kV})$ because the coupling is relatively low due to the large distance between the antenna and the plasma. 


\section{LOWER HYBRID HEATING}

In the lower hybrid (LH) heating domain, two waves coexist: the fast and the slow wave. For $N_{/ /}=0$, they are uncoupled and evanescent and only the FW exhibits a resonance at the LH. If $N_{/ /}$is increased (up to $N_{/ /} \approx 1$ ), both waves become propagating at the edge but merge some distance inside the plasma (confluence). Further in, both are evanescent (complex conjugate $k_{\perp}^{2}$ ) and the central part of the plasma is not accessible to the waves from the edge. It is only when the accessibility condition Eq.(65) of ref. $^{3}$, is satisfied that the two waves uncouple and propagate both inside the plasma. The accessibility condition implies that the launcher must be designed such as to launch waves with a parallel wavelength shorter than $\lambda_{/ /}=c /\left(N_{c} f\right)$

This is obtained by using an array of phased waveguides called the grill $^{21}$

The original concept of LH heating is to launch the slow wave (SW) at a frequency below the central value of the $\mathrm{LH}$ resonance. The $\mathrm{SW}$ then propagates up to the $\mathrm{LH}$ resonance and is absorbed there. It however became apparent from experiments that usually the wave tended to be absorbed by electron Landau damping before having reached the resonance. This is largely due to the cone propagation effect ${ }^{3}$. Indeed, the wave rays tend align to the magnetic field as the wave propagates inwards, eventually becoming exactly parallel at the LH resonance. Accordingly, the waves tend to circle around the plasma centre and to damp there. This efficient electron absorption is now exploited and recent LH heating experiments operate at frequencies above the central $\mathrm{LH}$ frequency, so that there is no longer a wave resonance inside the plasma and all the power goes to electrons. With an asymmetric $N_{/ /}$spectrum, LH heating is used as a current drive method. The cone behaviour is responsible for the main problem in applying $\mathrm{LH}$ to reactor-size machines, namely that the LH wave energy tends to propagate peripherally around the plasma and to deposit its energy away from the plasma centre. More precise information about the location where the power is deposited in toroidal geometry can be obtained using raytracing techniques ${ }^{22}$.

LH was first used as a heating method. The corresponding experimental results have been nicely summarised in a plot showing the different heating regimes in $\mathrm{FT}^{23}$. At low density, $\mathrm{LH}$ waves heat electrons. As the density is increased, electron heating fades away and ion heating sets in, creating ion tails. At still higher density, ion heating also decreases and disappears while parametric decay activity sets in. The different damping mechanisms of LH waves and the interpretation problems of experimental results, such as the spectral gap problem, are described in the book by Cairns ${ }^{24}$. Presently, the main application of LH is non-inductive current drive; it constitutes today the best, experimentally proven, current drive method. In large hot plasmas like ITER or reactors, the LH waves can usually not reach the centre. Accordingly, LH in ITER $^{25}$ is mainly seen as a tool for controlling the current profile by off-axis current drive in advanced scenarios ${ }^{26}$. It can also be used for saving voltseconds in the ramp-up phase of the plasma current.

\section{ALFVEN WAVE HEATING}

In the Alfvén wave domain, two types of cold-plasma waves can propagate ${ }^{3}$,

$\omega=k_{/ /} c_{\mathrm{A}}$ the shear Alfvén wave (SW)

$\omega=k c_{\mathrm{A}}$ the compressional Alfvén wave (FW)

The first of these relations implies that the shear Alfvén wave can propagate only along the field lines.

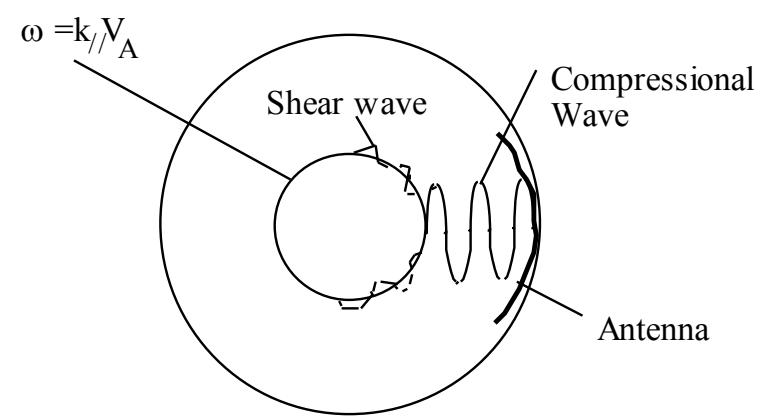

Fig. 3. The principle of Alfvén wave heating. Poloidal cross-section of the tokamak.

In an inhomogeneous plasma there is only one surface, close to a magnetic surface, where for a given $N_{/ /}$ the shear wave dispersion relation Eq.(33.1) is satisfied. So, the shear Alfvén wave can propagate only on that surface, as shown on Fig.3: it is trapped on that surface. Therefore, the idea is to launch from the outside the compressional Alfvén wave, which can propagate in all directions and reach the Alfvén resonance. Once the power is coupled to the shear wave, it stays on the magnetic surface and dissipates there. Note that the wavelength of the compressional wave is of the order of $1 \mathrm{~m}$. This means that, for $1 \mathrm{~m}$ wide or narrower antennas, most of the wave spectrum will be evanescent with an evanescence length of the order of the antenna size ${ }^{1}$.

From the experimental point of view the most extensive experiments and analysis of Alfvén wave heating have been performed on the TCA tokamak ${ }^{27}$ $\left(R_{0}=0.6 \mathrm{~m}\right)$. Although antenna coupling and general wave behaviour appeared to be in agreement with the theory, generally speaking little plasma heating was observed 
while the main effect of the RF was a large density increase, sometimes interpreted as an increase in the particle confinement time. In view of these disappointing results there have been few attempts to apply Alfvén wave heating to large tokamaks and this method is usually not mentioned for the heating of ITER or reactors. However, there has been some renewed interest in this field as the conversion to the kinetic Alfvén wave may induce poloidal shear flows, and possibly generate transport barriers ${ }^{28}$.

\section{FURTHER READING}

An excellent introductory overview of the different wave heating methods is given by Cairns ${ }^{24}$. A very complete work on plasma waves is that of Stix ${ }^{5}$. Brambilla $^{29}$ reviews the same subject with finer detail and a more direct view toward practical applications. In particular, many dispersion relation features are discussed in detail.

\section{REFERENCES}

1. R. KOCH, "The coupling of electromagnetic power to plasmas" These Proc.

2. P. LAMALLE, "Kinetic theory of plasma waves: Part III Inhomogeneous plasma" These Proc.

3. E. WESTERHOF, "Kinetic theory of plasma waves: Part II Homogeneous plasma" These Proc.

4. R. KOCH, "Heating by Waves in the Ion Cyclotron Frequency Range", Transactions of Fusion Technology, 33, 227, (1998)

5. T.H. STIX,"Waves in plasmas", AIP New York (1992).

6. ITER team, et al., "ITER Physics basis", Nucl. Fus. $\underline{39}(1999)$

7. V.P.BHATNAGAR, et al., "ICRF Heating and Synergistic LH and Fast-Wave current Drive in JET", 9th Top. Conf. on Radio Freq. Power in Plasmas, Charleston 115, (1991)

8. J.P.H.E. ONGENA, "Extrapolation to reactors", These Proc

9. J. JACQUINOT \& The JET Team, "Deuteriumtritium operation in magnetic confinement experiment: results and underlying physics", Plasma Phys. Contr. Fus. 41, A13,(1999)

10. K. THOMSEN, et al., "ITER H-mode confinement database update", Nucl. Fus. 34, 131, (1994).

11. R. KOCH, et al., "Progress on TEXTOR-94: Radiative-Improved-Mode and Radio-Frequency plasma production in the Ion Cyclotron Range", Proc. VI Ukrainian Conf. and School on Plasma Phys. and Contr. Fus., Alushta 1, 70,(1998)

12. L.-G. ERIKSSON, et al., "Theoretical analysis of ICRF heating in JET DT plasmas", ICPP \& 25th EPS
Conf. on Contr. Fus. and Plasma Phys., Prague, Europhysics Conf. Abstracts 22C, 1186, (1998)

13. L.-G. ERIKSSON, et al., "Comparison of Time Dependent Simulations with Experiments in Ion Cyclotron Heated Plasmas", Nucl. Fus., 33, 1037, (1993)

14. D.F.H. START, et al., "ICRF Results in D-T Plasmas in JET and TFTR and Implications for ITER", Plasma Phys. Contr. Fus., 40, A87, (1998)

15. R. KOCH et al., "A Comparison Between ICRF Theory and Experiment", Plasma Phys. Contr. Fus., 30, 1559, (1988).

16. M.PORKOLAB, et al., "Recent Progress in ICRF Physics", Plasma Phys. Contr. Fus., 40, A35,(1998)

17. R. KOCH et al., "Interaction of ICRF Waves with Fast Particles on TEXTOR", Plasma Phys. Contr. Fus., 37, A291, (1995).

18. V.P.BHATNAGAR, et al., "Local Magnetic Shear Control in a Tokamak via Fast Wave Minority Ion Current Drive: Theory and Experiments in JET", Nucl. Fus., 34, 1579, (1994)

19. R. KOCH et al., "Low loop voltage start-up of the TEXTOR-94 plasma with ICRF and/or NBI assistance", 26th EPS Conf. on Contr. Fus. \& Plasma Phys., Maastricht, (1999)

20. A. LYSSOIVAN et al., "ICRF Plasma Production in TORE SUPRA: Analysis of Antenna Coupling and Plasma Properties", ibid.

21. A. EKEDAHL, et al., "Conditioning and high power operation of the lower hybrid current drive launcher in JET" Report JET- P(97)19 (1997)

22. M. BRAMBILLA, "Ray tracing of lower hybrid and ion cyclotron waves", Computer Phys. Reports 4, 71 (1986).

23. F. ALLADIO et al., "Lower hybrid heating experiments on FT", in Proc. 4th Int. Symp. Heating in Toroidal Plasmas, (Roma), Ed. Knoepfel H., Sindoni E. 1, 546 (1984).

24. R.A. CAIRNS, "Radiofrequency heating of plasmas", Adam Hilger, IOP (1991).

25. P. FROISSARD, et al., "Lower hybrid wave injection system", NET report EUR FU/XII-218/113/98 (1998)

26. D. MOREAU et al., "ITER operation scenarios involving current profile control", NET report EUR FU/XII-218/112/98 (1998)

27. G. BESSON et al., "A review of Alfvén waves heating", Plasma Phys Contr. Fus. 28, 1291 (1986).

28. G. AMARANTE SECUNDO, et al., "Calculation of Alfvén wave driving forces, plasma flow, and current drive in the TCABR tokamak", 26th EPS Conf. on Contr. Fus. Plasma Phys., Maastricht, (1999)P3.092

29. M. BRAMBILLA, "Kinetic Theory of Plasma Waves - Homogeneous Plasmas", Clarendon Press Oxford (1998). 